Ann. Biol. anim. Bioch. Biophys., 1977, 17 (1), 95-105.

\title{
Estimation in vivo de la composition corporelle des agneaux à partir de l'espace de diffusion de l'eau lourde
}

\author{
par J. ROBELIN
}

avec la collaboration technique de Colette RAYNAL-RONGERE ef R. JAILLER

Laboratoire de la Production de Viande, I. N. R. A.

Theix, Saint-Genes-Champanelle, 63110 Beaumont

Summary. In vivo prediction of lamb body composition using heavy water space.

In this report, we study heavy water diffusion in 20 lambs 1, 5, 10 and 16 weeks old and the prediction of body fat by heavy water space.

Heavy water in plasma fluid is determined by infra-red spectrophotometry.

After intravenous injection, the heavy water diffuses rapidly into the total body water (4 hours). Its elimination rate is then regular (mean of 1.28 p. 100 per hour elapsed since injection), but varies greatly between animals (from 0.09 p. 100 to 4.21 p. 100 per hour).

Marker water space is a good indication of total body water volume of the animals $\left(R^{2}=0.996\right)$; with liveweight we can predict body fat weight quite accurately (coefficients of variation equal to 6.4 p. 100 and 10.5 p. 100 of this weight, depending on the age of the animal).

This accuracy is similar to that obtained in previous studies on lambs using tritiated water. The advantage of heavy water is that it is nor radioactive.

The accuracy of this prediction method is discussed in relation to different parameters : dosing of heavy water, methods of computing marker water space, water contained in the digestive tract.

\section{Introduction.}

L'éfude de la croissance et du développement des animaux nécessife la mesure de leur composition corporelle à différents stades. Parmi les différentes méthodes d'estimation in vivo de cette composition corporelle, nous avons relenu celle qui consiste à mesurer le volume hydrique des animaux à partir de l'espace de diffusion de l'eau marquée (cf. revue bibliographique de Robelin, 1973). Cette méthode a déjà fait l'objet de plusieurs travaux sur ovins, notamment ceux de Panaretto $(1963,1968)$, et de Searle (1970), ainsi que de premiers essais réalisés sur bovins dans notre Laboratoire par Geay (résultats non publiés). Le marqueur utilisé a toujours été l'eau tritiée à deux exceptions près (Foot et Greenhalgh, 1970 ; Trigg et al., 1973). Nous avons 
donc choisi d'utiliser l'eau lourde, marqueur non radioactif, posant moins de contraintes expérimentales, mais qui a l'inconvénient d'être plus difficile à doser que l'eau tritiée.

La présente étude rapporte les résultats que nous avons obtenus sur des agneaux, quant à la diffusion de l'eau lourde dans l'eau corporelle, sa vitesse de renouvellement dans l'organisme, et surtout quant à l'intérêt de son utilisation pour estimer la composition corporelle in vivo.

\section{Matériel et méthodes.}

Choix des animaux et conduife de l'expérimentation.

Cette étude a porté sur 20 agneaux mâles, âgés de 1, 5, 10 et 16 semaines qui ont été décrits précédemment (Robelin et al., sous presse). Les principales caractéristiques de ces animaux sont résumées au tableau 1.

\section{TABLEAU 1}

Age, poids ef composition chimique des agneaux

\begin{tabular}{|c|c|c|c|c|}
\hline 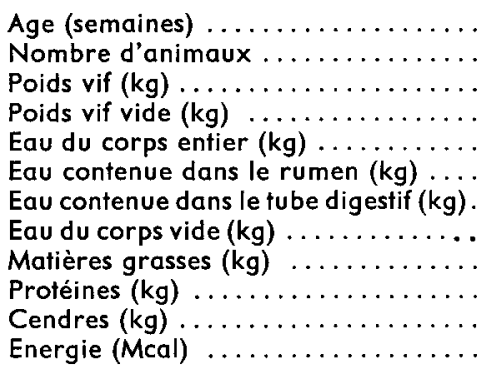 & $\begin{array}{c}1 \\
5 \\
5,25(0,43) \\
4,90(0,48) \\
3,92(0,31) \\
0,19(0,07) \\
0,30(0,08) \\
3,62(0,36) \\
0,31(0,05) \\
0,80(0,07) \\
0,17(0,02) \\
7,00(0,81)\end{array}$ & $\begin{array}{r}5 \\
5 \\
12,43(0,45) \\
11,49(0,64) \\
8,65(0,23) \\
0,58(0,14) \\
0,78(0,19) \\
7,87(0,36) \\
1,33(0,16) \\
1,92(0,13) \\
0,37(0,05) \\
22,83(2,05)\end{array}$ & $\begin{array}{c}10 \\
5 \\
22,43(1,97) \\
19,55(1,69) \\
15,33(1,18) \\
1,51(0,21) \\
2,37(0,39) \\
12,96(0,98) \\
2,44(0,57) \\
3,42(0,25) \\
0,73(0,04) \\
40,37(6,84)\end{array}$ & $\begin{array}{r}16 \\
5 \\
32,94(1,48) \\
28,77(1,36) \\
22,01(1,19) \\
2,57(0,37) \\
3,44(0,47) \\
18,57(1,11) \\
4,08(0.29) \\
4,99(0,26) \\
1,13(0,04) \\
64,15(1,88)\end{array}$ \\
\hline
\end{tabular}

Les nombres entre parenthèses sont les écarts types.

Après pesée des animaux, l'eau lourde a été injectée à l'aide d'un cathéter posé sur la veine jugulaire, et relié à deux seringues par une vanne «trois voies ». Ces deux seringues contenaient respectivement du $D_{2} \mathrm{O}$, dont la quantité injectée a été mesurée par double pesée, et du soluté isotonique de $\mathrm{NaCl}$ destiné au lavage de la vanne et du cathéter. Nous avons injecté une quantité voisine de $0,6 \mathrm{~g}$ par $\mathrm{kg}$ de poids vif, qui correspond à une concentration du marqueur dans l'eau corporelle voisine de 1 p. 1000.

Nous avons prélevé des échantillons de $3 \mathrm{ml}$ de sang, $10 \mathrm{mn}, 20 \mathrm{mn}, 2 \mathrm{~h}, 4 \mathrm{~h}$ et $6 \mathrm{~h}$ après l'injection, ainsi qu'au moment de l'abattage des animaux, qui a eu lieu $12 \mathrm{~h}$ après l'injection. L'eau ef les aliments ont été laissés à la disposition des animaux pendant toute la période de prélèvement. L'eau des échantillons a été recueillie par une lyophilisation de $5 \mathrm{~h}$, stockée dans des seringues de $1 \mathrm{ml}$, hermétiquement fermées, 
el conservées à la température de $4^{\circ} \mathrm{C}$. Après l'abattage, la composition chimique des animaux a été mesurée par broyage du corps entier et analyse chimique selon la méthode décrite précédemment (Robelin et al, sous presse).

\section{Méthode de dosage de l'eau lourde.}

Parmi les méthodes utilisables : mesure du point de congélation du mélange eau/eau lourde (Reaser et Burch, 1958), mesure de la densité du mélange eau/eau lourde (Keston et al., 1937 ; Schloerb ef al., 1961), dosage par spectrographie de masse (Thomas, 1950 ; Washburn et al., 1953 ; Arnett et al., 1963 ; Bricout et Merlivat, 1971), dosage par spectrophotométrie infrarouge (Thornton et Condon, 1950 ; Gaunt, 1954 ; Berglund-Larsson, 1956 ; Turner et al., 1960 ; Foot et Greenhalgh, 1970), dosage par chromatographie en phase gazeuse (Mendez et al., 1970), la plus précise est la mesure par spectrographie de masse. Cependant, son coût élevé nous a conduit à choisir la mesure par spectrophotométrie infrarouge, qui présente le plus d'avantages d'après la plupart des travaux cités ci-dessus et d'après une étude comparative effectuée par Graystone et al. (1967). Nous avons effectué les mesures avec un appareil Leiłz (modèle $3 \mathrm{G}$ ).

Le dosage consiste à mesurer l'absorption de la lumière infrarouge de longueur d'onde 3,98 $\mu$ (Thornton et Condon, 1950 ; Berglund-Larsson, 1956), par un échantillon du mélange eau/eau lourde contenu dans une cuve d'épaisseur fixée. Dans une zone de concentration en eau lourde voisine de 1 p. 1000 , nous avons obtenu une précision maximale pour une épaisseur de cuve de $0,1 \mathrm{~mm}$.

Nous avons mesuré la concentration en eau lourde de nos échantillons, par la méthode d'intrapolation décrite par Foot et Greenhalgh (1970) :

$$
X=C_{1}+\left(T-T_{1}\right)\left(\frac{T_{2}-T_{1}}{C_{2}-C_{1}}\right) \text {. }
$$

$T_{1}$ et $T_{2}$ sont les transmissions (enregistrées par le spectrophotomètre), à travers deux échantillons témoins de concentrations $C_{1}$ et $C_{2}$, connues exactement. $T$ est la transmission enregistrée correspondanł à l'échantillon dont on veut mesurer la concentration $X$. Des éfudes préliminaires nous ont montré que l'écart-type de la mesure était égal à 6,46 p. 100 de la concentration mesurée; nous avons donc effectué 6 mesures par échantillon, de façon à obtenir une précision suffisante sur la moyenne :

$$
\frac{6,46 \text { p. } 100}{\sqrt{6}}=2,6 \text { p. } 100 .
$$

Calcul de l'espace de diffusion de l'eau lourde.

Après l'injection, la concentration $(C)$ en eau lourde dans l'eau plasmatique, suit en fonction du temps ( $t$ ), une courbe biphasique qui peut être ajustée à une somme de deux exponentielles :

$$
C=A_{1} \times 10^{-a_{1} t}+A_{2} \times 10^{-a_{2} t} .
$$

En admettant le schéma de diffusion adopté par Fayet (1971), comprenant deux compartiments, et en suivant le raisonnement développé par Aubert et Milhaud (1960), 
relatif à un modèle à plusieurs compartiments ouverts, on peut calculer les volumes respectifs des deux compartiments (VI et VE), la vitesse de transfert (VTF) de l'eau entre ces deux espaces, et la vitesse totale de sortie (VTS) de l'eau de l'organisme ; soit $\mathrm{QI}$, la quantité de marqueur injectée, et $A_{1}, a_{1}, A_{2}$, $a_{2}$, les coefficients de la courbe de diffusion (calculés expérimentalement) :

$$
\begin{aligned}
V E & =\frac{Q I}{A_{1}+A_{2}} \\
V T S & =Q I /\left(\frac{A_{1}}{a_{1}}+\frac{A_{2}}{a_{2}}\right) . \\
V T F & =\frac{\left(A_{1} a_{1}+A_{2} a_{2}\right) Q I-V T S}{\left(A_{1}+A_{2}\right)^{2}} . \\
V I & =V T F \times \frac{A_{1}+a_{2}}{A_{1} a_{2}+A_{2} a_{1}} .
\end{aligned}
$$

Le volume hydrique total $V$ est égal à :

$$
\mathrm{V}=\mathrm{VI}+\mathrm{VE} \text {. }
$$

Nous avons également calculé le volume hydrique total par une méthode simplifiée, consistant à extrapoler au temps zéro, la seconde exponentielle $\left(A_{2} 10^{-a_{2} t}\right)$. On obtient ainsi la concentration initiale théorique $\left(A_{2}\right)$, correspondant à la dilution immédiate du marqueur dans l'ensemble du volume hydrique ; ce dernier est alors égal à :

$$
\mathrm{V}=\frac{\mathrm{Q} \mathrm{I}}{\mathrm{A}_{\mathbf{2}}} \text {. }
$$

\section{Résultats.}

Evolution de la concentration en eau lourde de l'eau plasmatique après l'injection.

La courbe moyenne de diffusion du marqueur sur l'ensemble des 20 animaux a été la suivante :

$$
\frac{\mathrm{C}}{\mathrm{Co}}=0,35410^{-0,42 \mathrm{t}}+10^{-0,0058 \mathrm{t}}
$$

dans laquelle Co est la concentration initiale théorique, variable entre animaux en fonction de la quantité de marqueur injectée.

L'eau lourde a diffusé très rapidement dans l'eau corporelle ; dix minutes après l'injection, sa concentration dans l'eau plasmatique n'était égale qu'à 1,30 fois la concentration théorique $(\mathrm{Co})$ correspondant à une diffusion instantanée. Ensuite, la concentration a diminué plus lentement. L'évolution est devenue linéaire en coordonnées semi-logarithmiques à partir de $2 \mathrm{~h}$ après l'injection chez les animaux âgés de 1 et 16 semaines, et à partir de $4 \mathrm{~h}$ chez les animaux âgés de 5 et 10 semaines. L'équilibre du marqueur dans l'eau corporelle a donc été atteint au plus tard en $4 \mathrm{~h}$, durée comparable à celle qui a été trouvée par différents auteurs avec l'eau tritiée chez l'agneau (Searle, 1970 ; Smith et Sykes, 1974).

Dès lors, la concentration du marqueur a diminué régulièrement de 1,28 p. 100 de sa valeur par heure écoulée depuis l'injection (moyenne calculée sur l'ensemble 
des 20 animaux), ce qui correspond à une demi-vie moyenne de $54 \mathrm{~h}$. La vitesse d'élimination a été très variable entre animaux (de 0,09 p. 100 à 4,71 p. 100 par heure) ; c'est pourquoi, les valeurs moyennes observées sur chacun des groupes d'animaux $(1,24$ p. $100 ; 1,15$ p. $100 ; 1,68$ p. 100 et 1,08 p. 100 respectivement chez les agneaux âgés de $1,5,10$ et 16 semaines) ne sont pas significativement différentes entre elles.

Nous avons calculé la concentration initiale théorique du marqueur en extrapolant au temps zéro, la droite correspondant à la disparition du marqueur entre 4 ef $12 \mathrm{~h}$ après l'injection. L'erreur inhérente à la détermination de cette droite (erreur de mesure de la concentration des échantillons, non-linéarité des trois points correspondant aux temps 4,6 et 12 h), a entraîné théoriquement une erreur relative sur la mesure du volume de diffusion de l'eau lourde, égale à 4,66 p. 100 de ce volume.

Le calcul du volume hydrique par la méthode la plus élaborée a donné une estimation systématiquement inférieure de 1,03 p. $100(0,7$ à 1,1 p. 100) à l'estimation précédente.

TABLEAU 2

Comparaison entre les poids d'eau et de matières grasses, mesurés par analyse chimique et estimés à partir de l'espace de diffusion de l'equ lourde

\begin{tabular}{|c|c|c|c|c|c|c|c|}
\hline \multicolumn{4}{|c|}{ Agneaux âgés de 1 et 5 semaines } & \multicolumn{4}{|c|}{ Agneaux âgés de 10 et 16 semaines } \\
\hline \multicolumn{2}{|c|}{ Poids d'equ (g) } & \multicolumn{2}{|c|}{$\begin{array}{l}\text { Poids des } \\
\text { matières grasses }(g)\end{array}$} & \multicolumn{2}{|c|}{ Poids d'eau $(\mathrm{g})$} & \multicolumn{2}{|c|}{$\begin{array}{c}\text { Poids des } \\
\text { matières grasses (g) }\end{array}$} \\
\hline Réel & Estimé & Réel & Estimé & Réel & Estimé & Réel & Estimé \\
\hline $\begin{array}{l}3953 \\
3851 \\
3468 \\
4340 \\
4006 \\
8920 \\
8638 \\
8657 \\
8748 \\
8289\end{array}$ & $\begin{array}{l}4219 \\
3926 \\
3357 \\
4802 \\
4110 \\
8592 \\
8364 \\
7945 \\
8821 \\
7968\end{array}$ & $\begin{array}{r}276 \\
280 \\
285 \\
398 \\
295 \\
1392 \\
1224 \\
1416 \\
1499 \\
1098\end{array}$ & $\begin{array}{r}283 \\
293 \\
274 \\
386 \\
292 \\
1411 \\
1139 \\
1396 \\
1433 \\
1237\end{array}$ & $\begin{array}{l}14851 \\
16886 \\
16277 \\
14206 \\
14440 \\
21064 \\
22517 \\
21563 \\
23871 \\
21058\end{array}$ & $\begin{array}{l}14715 \\
16957 \\
15884 \\
14830 \\
14830 \\
21131 \\
23833 \\
21461 \\
23803 \\
20649\end{array}$ & $\begin{array}{ll}2 & 067 \\
2 & 675 \\
3 & 241 \\
1 & 762 \\
2 & 450 \\
4 & 400 \\
3 & 911 \\
3 & 674 \\
4 & 123 \\
4 & 291\end{array}$ & $\begin{array}{l}2241 \\
2673 \\
2944 \\
1948 \\
2148 \\
4337 \\
3630 \\
4118 \\
4396 \\
4085\end{array}$ \\
\hline
\end{tabular}

Estimation de l'eau corporelle à partir de l'espace de diffusion de l'eau lourde.

Nous avons mis en parallèle, dans le tableau 2, pour chaque animal, le poids d'eau du corps entier (contenu digestif inclus) mesuré par dessiccation ( $Y$ ) et celui estimé à partir de l'espace de diffusion de l'eau lourde $(X)$. La relation entre $Y$ et $X$, exprimée en grammes, est la suivante :

$$
\mathrm{Y}=0,982 \mathrm{X}+163 \quad \mathrm{R}^{2}=0,996 \quad \text { SyX }=416(\mathrm{~g}) \quad \mathrm{n}=20 .
$$

L'écart-type résiduel, égal à $416 \mathrm{~g}$ pour l'ensemble des animaux, est variable avec l'âge des agneaux ou le poids d'eau corporelle : $280 \mathrm{~g}, 446 \mathrm{~g}, 424 \mathrm{~g}, 693 \mathrm{~g}$ respectivement chez les agneaux âgés de $1,5,10$ et 16 semaines, soit 7,1 p. 100, 5,2 p. 100 , 
2,8 p. 100 et 3,1 p. 100 du poids d'eau. L'espace de diffusion de l'eau lourde est donc un bon estimateur de l'eau totale du corps entier

La liaison entre les deux valeurs après transformation logarithmique n'est pas plus élevée $\left(R^{2}=0,996\right)$; l'écart-type de cette nouvelle régression (log $Y=0,9910 \log$ $X+0,01078$ ) est égal à 4,4 p. 100 de Y. Cette précision est du même ordre de grandeur que les valeurs trouvées chez les agneaux par différents auteurs avec l'eau lourde (Foot ef Greenhalgh, 1970) ou avec l'eau tritiée (cf. revue bibliographique de Robelin, 1973 ; Donnely et Freer, 1974 ; Sikes, 1974).

Estimation des poids des consfituants chimiques du corps vide.

Indépendamment de l'âge des animaux, le poids des différents constituants chimiques est fortement lié au poids vif ( $R^{2}$ supérieur à 0,99$)$ (†abl. 3) ; ce sont les matières grasses qui ont le coefficient de variation le plus élevé (CVR $=11,4$ p. 100).

\section{TABLEAU 3}

Relations entre le poids des différents constituants chimiques du corps vide (Y), le poids vif (PV) et le volume de diffusion de l'eau lourde (VD) chez l'agneau

\begin{tabular}{|c|c|c|c|c|c|}
\hline \multirow{3}{*}{$\begin{array}{l}\text { Variables } \\
\text { dépendantes } \\
\text { (Y) }\end{array}$} & \multicolumn{4}{|c|}{ Variables indépendantes } & \multirow{3}{*}{$\begin{array}{c}\text { Valeur } \\
\text { du test de } F \\
\text { sur la différence } \\
R 2^{2}-\mathrm{R} 1^{2}\end{array}$} \\
\hline & \multicolumn{2}{|c|}{ Poids vif } & \multicolumn{2}{|c|}{$\begin{array}{l}\text { Poids vif et espace } \\
\text { de diffusion de } \mathrm{D}_{2} \mathrm{O}\end{array}$} & \\
\hline & $\mathrm{R} 1^{2}$ & $\begin{array}{c}\text { CVR } 1 \\
\text { p. } 100 \text { de Y }\end{array}$ & $\mathrm{R} 2^{2}$ & $\begin{array}{c}\text { CVR } 2 \\
\text { p. } 100 \text { de } Y\end{array}$ & \\
\hline $\begin{array}{l}\text { Eau } \ldots \ldots \ldots \ldots \\
\text { Matières grasses } \ldots \ldots \\
\text { Cendres } \ldots \ldots \ldots \ldots \ldots \\
\text { Protéines } \ldots \ldots \ldots \ldots \ldots \\
\text { Energie } \ldots \ldots \ldots \ldots \ldots\end{array}$ & $\begin{array}{l}0,9980 \\
0,9908 \\
0,9913 \\
0,9982 \\
0,9964\end{array}$ & $\begin{array}{r}3,0 \\
11,4 \\
7,9 \\
3,5 \\
5,7\end{array}$ & $\begin{array}{c}X \\
0,9952 \\
0,9914 \\
0,9982 \\
0,9984\end{array}$ & $\begin{array}{c}X \\
8,4 \\
7,9 \\
3,5 \\
4,0\end{array}$ & $\begin{array}{c}x \\
12,83 \\
0,12(\mathrm{NS}) \\
0,53(\mathrm{NS}) \\
9,09\end{array}$ \\
\hline
\end{tabular}

Les relations ont été calculées à partir du logarithme décimal des poids Y, PV ef VD (exprimés en $\mathrm{kg}$ ) à l'intérieur de chacun des $\mathbf{4}$ groupes d'animaux.

$\mathrm{R}^{1}{ }^{1}$ ef $\mathrm{R} 2^{2}$ sont les carrés des coefficients de corrélation simple ou multiple des deux relations entre $Y$ et le poids vif ou entre $Y$, le poids vif et le volume de diffusion de l'eau lourde.

CVR 1 et CVR 2 sont les coefficients de variation résiduels, écarts-types résiduels respectifs de ces deux relations, exprimés en pourcentage de $Y$.

Le niveau de signification du volume de diffusion de l'eau lourde dans la seconde relation a été testé sur la différence $R 2^{2}-R 1^{2}$.

La mesure de l'espace de diffusion de l'eau lourde permet d'améliorer significativement $(P<0,01)$ l'estimation du poids matières grasses ef de la valeur calorifique à partir du poids vif, mais pas celle du poids des protéines ou des minéraux (tabl. 3). Le coefficient de variation résiduel des matières grasses passe de 11,4 p. 100 avec le poids vif seul, à 8,4 p. 100 avec le poids vif et l'espace de diffusion de l'eau lourde, 7,5 p. 100 avec le poids vif et l'eau totale réelle, et 6,9 p. 100 avec le poids vif vide et l'eau du corps vide. 
L'âge des animaux a une légère influence ( $P$ compris entre 0,05 et 0,10 ) sur les relations d'estimation des matières grasses et de l'énergie, à partir du poids vif et de l'espace de diffusion de l'eau lourde ou sur l'équation d'estimation des protéines, à partir du poids vif seul. On peut séparer les animaux en deux populations significativement différentes : les agneaux âgés de 1 à 5 semaines d'une part et ceux de 10 et 16 semaines d'autre part, soit respectivement les animaux recevant du lait et ceux qui avaient été sevrés à l'âge de 6 semaines.

Les coefficients des équations, correspondant à chacun des deux groupes d'animaux, figurent au tableau 4. Les coefficients de variation résiduels des matières grasses

TABLEAU 4

Equations d'estimation des principoux composants chimiques ef de la valeur calorifique du corps entier à partir du poids vif (PV) et de l'espace de diffusion de l'eau lourde (ED) (1)

\begin{tabular}{|c|c|c|c|c|c|}
\hline \multirow[b]{2}{*}{$\begin{array}{l}\text { Composants } \\
\text { estimés }\end{array}$} & \multicolumn{5}{|c|}{ Coefficients des équations } \\
\hline & $\begin{array}{c}\text { Terme } \\
\text { constant } \\
\text { (BO) }\end{array}$ & $\begin{array}{l}\text { Coefficient } \\
\text { de PV } \\
\text { (B1) }\end{array}$ & $\begin{array}{l}\text { Coefficient } \\
\text { de ED } \\
\text { (B2) }\end{array}$ & $\begin{array}{c}\text { CVR } 1 \\
(2) \\
\text { (p. 100) }\end{array}$ & $\begin{array}{c}\text { CVR } 2 \\
(2) \\
\text { (p. 100) }\end{array}$ \\
\hline $\begin{array}{c}\text { Matières I (3) } \ldots \ldots \ldots \ldots \ldots \ldots \\
\text { grasses II (3) } \ldots \ldots \ldots \ldots \ldots\end{array}$ & $\begin{array}{l}-1,7627 \\
-1,8664\end{array}$ & $\begin{array}{l}3,546 \\
3,727\end{array}$ & $\begin{array}{l}-2,152 \\
-2,364\end{array}$ & $\begin{array}{r}6,4 \\
10,5\end{array}$ & $\begin{array}{r}9,4 \\
14,6\end{array}$ \\
\hline Protéines $\begin{aligned} & 1 \\
& \end{aligned}$ & $\begin{array}{r}-0,8334 \\
-0,7745\end{array}$ & $\begin{array}{l}1,0210 \\
0,9692\end{array}$ & $\begin{array}{l}0 \\
0\end{array}$ & - & $\begin{array}{l}3,8 \\
2,4\end{array}$ \\
\hline Energie $\begin{aligned} & \ldots \ldots \ldots \ldots \ldots \ldots \ldots \\
& \| \ldots \ldots \ldots \ldots \ldots\end{aligned}$ & $\begin{array}{r}-0,1543 \\
-0,2862\end{array}$ & $\begin{array}{l}2,3055 \\
2,6597\end{array}$ & $\begin{array}{r}-1,0893 \\
-1,4443\end{array}$ & $\begin{array}{l}2,7 \\
4,2\end{array}$ & $\begin{array}{l}4,3 \\
6,3\end{array}$ \\
\hline
\end{tabular}

(1) Les relations ont été établies après transformation en logarithmes décimaux des différents poids exprimés en $\mathrm{kg}$ et de la valeur calorifique exprimée en $\mathrm{kcal}$ :

$$
\log Y=B_{0}+B_{1} \log P V+B_{2} \log E D \text {. }
$$

(2) CVR 1 et CVR 2 sont coefficients de variation résiduels des équations ne faisant intervenir que le poids vif (CVR 2) ou le poids vif et l'espace de diffusion de l'eau lourde (CVR 1).

(3) Les équations I ef II se rapportent respectivement aux agneaux âgés de 1 à 5 semaines et aux agneaux âgés de 10 à 16 semaines.

et de l'énergie sont plus faibles chez les animaux les plus jeunes (respectivement 6,4 et 2,7 p. 100) que chez les agneaux plus âgés (respectivement 10,5 et 4,2 p. 100). Ces précisions, voisines de 8 p. 100 pour les matières grasses, et 3 p. 100 pour l'énergie, sont comparables à celles qui ont été obtenues sur agneaux avec l'eau tritiée (Searle, 1970 ; Farrel et Reardon, 1971 ; Donnely et Freer, 1974 ; Smith et Sykes, 1974) ou avec l'eau lourde (Foot et Greenhalgh, 1970 ; Trigg ef al., 1973).

La mesure de l'eau contenue dans le rumen, fortement liée $(R=0,96)$ à l'eau contenue dans l'ensemble du tube digestif, a permis d'améliorer significativement la précision de l'estimation des matières grasses chez les animaux âgés de 10 à 16 semaines. Le coefficient de variation résiduel est passé de 10,5 à 7,9 p. 100. 


\section{Discussion.}

L'ensemble de nos résultats confirme, dans le cas de l'eau lourde, ceux qui ont été obtenus précédemment chez l'agneau avec l'eau tritiée, tant sur le plan de la diffusion du marqueur et de sa vitesse d'élimination (Morris et al., 1962 ; Searle, 1970 ; Smith et Sykes, 1974), que sur le plan de la précision de l'estimation du volume hydrique ef de sa liaison avec le poids des matières grasses corporelles (Searle, 1970 ; Farrel et Reardon, 1971 ; Donnely ef Freer, 1974 ; Smith et Sykes, 1974). L'eau lourde présente les mêmes caractéristiques de diffusion que l'eau tritiée, mais elle possède l'avantage de ne pas être radioactive.

Elle permet d'estimer in vivo le poids des matières grasses corporelles avec une bonne précision ( $C V R \leqslant 10$ p. 100) ; cette dernière dépend de plusieurs facteurs dont les plus importants sont la précision du dosage de l'eau lourde, la méthode de détermination de l'espace de diffusion du marqueur et la variabilité de la quantité d'eau contenue dans le tube digestif.

En effectuant 6 déterminations par échantillon, nous avons mesuré avec une bonne précision (2,6 p. 100) la concentration en eau lourde dans l'eau plasmatique ; cette précision est comparable à ce que d'autres auteurs ont trouvé avec cette méthode de dosage par spectrophotométrie infrarouge (Thornton et Condon, 1950 ; Gaunt, 1954). Nous nous efforçons actuellement d'améliorer la technique, de façon à diminuer le nombre de déterminations à réaliser.

Nous avons utilisé deux méthodes de calcul du volume de diffusion de l'eau lourde : calcul à partir des coefficients $A_{1}, a_{1}, A_{2}, a_{2}$ de la courbe de diffusion, ou calcul à partir de $A_{2}$ seulement. Nous avons observé une très faible différence entre ces deux méthodes de calcul ( 1 p. 100 du volume hydrique) : d'un point de vue théorique, cette différence de précision ne provient que des coefficients $A_{1}$ et $a_{1}$ (début de la courbe de diffusion). L'importance de cette différence ne justifie pas le nombre de mesures supplémentaires à effectuer pour calculer ces coefficients $A_{1}$ et $a_{1}$. Inversement, pour un nombre de mesures fixé, il est plus judicieux d'effectuer ces mesures après l'équilibre du marqueur, de façon à améliorer la précision de l'estimation de $A_{2}$. $A u$ cours de la plupart des travaux effectués jusqu'à présent (Robelin, 1973 ; Donnely et Freer, 1974), l'espace de diffusion a été calculé par une méthode encore plus simple : rapport entre la quantité de marqueur injecté et la concentration du marqueur après l'équilibre. Cette dernière méthode nous semble moins précise que les précédentes ; en effet, elle ne tient pas compte de la vitesse d'élimination du marqueur qui a été très variable chez nos animaux (de 0,09 à 4,71 p. 100 par heure).

Nos résultats ont mis en évidence une influence de l'âge et du régime alimentaire des animaux sur les relations d'estimation du poids des matières grasses ou de la valeur calorifique, ce qui nous a conduit à proposer deux équations d'estimation. Ce fait peut être lié en partie à la diminution de la teneur en eau du corps délipidé (poids vif vide-poids des matières grasses), phénomène déjà décrit, par Reid et al. (1968) notamment, et que nous avons observé également avec nos animaux; aux différents âges de $1,5,10$ et 16 semaines, la teneur en eau du corps délipidé a été égale respectivement à 78,$8 ; 77,4 ; 75,7$ et 75,2 p. 100 . Cependant, il est probable que l'influence de l'âge sur la relation d'estimation est due en majeure partie au fait que les animaux 
sont passés d'une alimentation lactée à une alimentation à base de foin et d'aliments concentrés. Ce changement a été accompagné d'une augmentation de la part de l'eau totale contenue dans le tube digestif (15,5 p. 100 au lieu de 8,6 p. 100), ce qui a eu une influence sur la relation entre l'eau corporelle totale et le poids des matières grasses ou la valeur énergétique.

Nous avons observé que la mesure de l'eau contenue dans le rumen, permettait d'améliorer sensiblement la précision de l'estimation du poids des matières grasses chez les animaux âgés de 10 à 16 semaines. Il serait donc intéressant de disposer d'une méthode précise, permettant d'estimer l'eau contenue dans le rumen (ou le tube digestif total) ; à défaut d'une telle méthode, il est nécessaire de standardiser au maximum les conditions d'alimentation des animaux pendant la mesure de l'espace de diffusion de l'eau lourde, afin de réduire la variabilité du poids de l'eau contenue dans le tube digestif. Ces conditions prennent une importance plus grande encore si on veut utiliser cette méthode d'estimation des lipides chez les bovins; en effet, la part de l'eau totale contenue dans le tube digestif peut varier de 17 p. 100 à 28 p. 100 selon le régime alimentaire (calcul effectué à partir de résultats obtenus par Beranger et al.).

En conclusion, la précision que nous avons obtenue avec cette méthode d'estimation in vivo voisine de 8 p. 100 pour les matières grasses, de 3 à 4 p. 100 pour les protéines ef l'énergie, est bonne. Elle est comparable à celle que nous avons obtenue par ailleurs chez les bovins (Robelin et Geay, 1975), à partir de la dissection d'un morceau représentatif de la carcasse prélevé après l'abattage. Elle dépend surtout de la précision du dosage de l'eau lourde dans l'eau, qui peut être améliorée, mais aussi de la variabilité du poids de l'eau contenue dans le tube digestif.

Cetfe méthode d'estimation in vivo de la composition corporelle permet de décrire l'évolution de la lipogénèse chez le même animal, dans les limites d'âge et de poids correspondant au calcul des équations d'estimation. Les équations peuvent servir également à constituer des lots d'animaux comparables au début d'une expérience.

Compte tenu de ces résultats, nous poursuivons maintenant nos travaux sur des jeunes bovins en croissance, pour lesquels l'intérêt de cette estimation indirecte est rendue plus importante encore par le coût très élevé de l'abattage et de l'analyse chimique directe du corps entier. Enfin, cette méthode pourrait également être utilisée pour décrire les variations des réserves lipidiques d'animaux en gestation ou en lactation chez lesquels la composition corporelle n'est certainement pas reliée étroitement au poids vif.

Accepté en aoat 1976.

Remerciements. - Cette étude a été réalisée grâce à l'aide de la D. G. R. S. T. (convention no 72.7.07.39) à qui nous exprimons nos remerciements. Nous tenons également à remercier le Centre de Mesures Physiques de l'Université de ClermontFerrand (Madame M. Tissier) qui a mis gracieusement à notre disposition le spectrophotomètre infrarouge que nous avons utilisé. 


\section{Références}

AUBERT J. P., MILHAUD G., 1960. Méthode de mesure des principales voies du métabolisme calcique chez l'homme. Biochim. Biophys. Acta, 39, 122-139.

BERGLUND-LARSSON U., 1956. Determination of small amounts of deuterium oxide in water by infra-red spectroscopy. Acta. Chem. Scand. 10, 701-702.

BRICOUT J., MERLIVAT L., 1971. Sur la teneur en deutérium des jus d'oranges. C. R. Acad. Sc. Paris, Sér. D, 273, 1021-1023.

DONNELY J. R., FREER M., 1974. Prediction of body composition in live sheep. Aust. J. Agric. Res., 25, 825-834.

FARRELL D. J., REARDON T. F., 1972. Undernutrition in grazing sheep. III. Body composition and its estimation in vivo. Aust. J. Agric. Res., 23, 511-517.

FAYET J. C., 1971. Plasma and faecal osmolality, water kinetics and body fluid compartments in neonatal calves with diarrhoea. Br. Vet. J. 127, 37-44.

FOOT J. Z., GREENHALGH J. F. D., 1970. The use of deuterium oxide space to determine the amount of body fat in pregnant black-face ewes. Br. J. Nutr. 24, 815-825.

GAUNT J., 1964. The determination of deuterium oxide by infra-red spectrometry. Analyst, 79, 580-585.

GRAYSTONE J., SEITCHIK J., MILCH R., SHULMAN G. P., CHEEL D. B., 1967. Measurement of $\mathrm{D}_{2} \mathrm{O}$ in plasma water by freezing point elevation, falling drop, infra-red absorption and gas chromatography. J. Lab. Clin. Med., 69, 885-892.

KESTON A. S., RITTENBERG D., SCHOENHEIMER R., 1937. Determination of deuterium oxide in compounds. J. Biol. Chem. 122, 227-237.

MENDEZ J., PROKOP E., PICON-REATEGUI E., AKERS R., BUSHKIRK E. R., 1970. Total body water by $\mathrm{D}_{2} \mathrm{O}$ dilution using saliva samples and gas chromatography. J. Appl. Physiol., 28, 354-357.

MORRIS R. J. H., HOWARD B., MacFARLANE W. V., 1962. Interaction of nutrition and air temperature with water metabolism of Merino wethers shorn in winter. Aust. J. Agric. Res., 13, 320-334.

PANARETTO B. A., 1963. Body composition in vivo. III. The composition of living ruminants and its relation to the tritiated water spaces. Austr. J. Agric. Res. 14, 944-952.

PANARETTO B. A., 1968. Body composition in vivo. IX. The relation of body composition to the tritiated water spaces of ewes and wethers fasted for short periods. Aust. J. Agric. Res., 19, 267-272.

REASER P. B., BURCH G. E., 1958. Determination of deuterium oxide in water by measurement of freezing-point. Science, 128, 445-446.

REID J. T., BENSADOUN A., BULL L. S., BURTON J. H., GLEESON P. A., HAN I. K., JOO Y. D., JOHNSON D. E., MacMANUS W. R., PALADINES O. L., STROUD J. W., TYRREL H. F., VAN NIEKERK B. D. H., WELLINGTON G. H., WOOD J. D., 1968. Changes in body composition and meat characteristics accompanying growth of animals. Proc. Cornell Conf. Feed. Manuf., 18, 37.

ROBELIN J., 1973. Revue bibliographique : Estimation de la composition corporelle des animaux à partir des espaces de diffusion de l'eau marquée. Ann. Biol. anim. Bioch. Biophys., 13, 285-305.

ROBELIN J., THERIEZ M., ARNAL M., FERRARA M. Ann. Zootech. (sous presse).

ROBELIN J., GEAY Y., BERANGER C., 1975. Estimation de la composition chimique des carcasses de jeunes bovins mâles, à partir de la proportion de dépôts adipeux d'un morceau monocostal prélevé au niveau de la «11e côte ». Ann. Zootech., 24, 323-326. 
SEARLE T. W., 1970. Body composition in lambs and young sheep and its prediction in vivo from tritiated water space and body weight. J. agric. Sci., 74, 357-362.

SCHLOERB P. R., FRUS-HANSEN B. J., EDELMAN I. S., SHELDON D. B., MOORE F. D., 1951. The measurement of deuterium oxide in body fluids by the falling drop method. Lab. Clin. Med., 37, 652-653.

SMITH B. S. W., SYKES A. R., 1974. The effect of route of dosing and method of estimation of tritiated water space on the determination of total body water and prediction of body fat in sheep. J. agric. Sci., 82, 105-112.

SYKES A. R., 1974. The prediction of body composition of hill sineep from body weight, red cell volume, and tritiated water space. J. agric. Sci., 82, 269-275.

THOMAS B. W., 1960. Determination of heavy water by mass spectrometer. Anal. Chem., 22, 14761478.

THORNTON V., CONDON F. E., 1950. Infra-red spectrometric determination of deuterium oxide in water. Anal. Chem. 22, 690-691.

TRIGG T. E., DOMINGO E. A., TOPPS J. H., 1973. Estimation of body composition of sheep by isotope dilution techniques. 2. Deuterium oxide and tritiated water. Proc. Nut. Soc., 32, 21 A.

TURNER M. D., NEELY W. A., HARDY J. D., 1960. Rapid determination of deuterium oxide in biological fluids. J. Appl. Physiol. 15, 309-310.

WASHBURN H. W., BERRY C. E., HALL L. G., 1953. Measurement of deuterium oxide concentration in water samples by the mass spectrometer. Anal. Chem., 25, 130-134. 